PROCEEDINGS OF THE

AMERICAN MATHEMATICAL SOCIETY

Volume 126, Number 4, April 1998, Pages 1143-1148

S 0002-9939(98)04178-1

\title{
ENSEMBLES SUR LESQUELS LES POLYNÔMES SONT DÉTERMINÉS PAR LEUR IMAGE
}

\author{
MICHEL SAVOYANT
}

(Communicated by Albert Baernstein II)

\begin{abstract}
Let $A$ be a non-empty subset of the complex plane $\mathbb{C}$, and $P, Q$ two complex polynomials. If $P$ and $Q$ having the same image on $A$ implies $P=Q$, we say that $A$ is a generalized unicity set (for polynomials). We construct in this paper a subset $A$ of $\mathbb{C}$ such that $A$ and $\mathbb{C} \backslash A$ are generalized unicity sets, and we give an example of a generalized unicity set which is open, connected and unbounded.

RÉsumÉ. Soit $A$ un sous-ensemble non vide du plan complexe $\mathbb{C}$, et $P, Q$ deux fonctions polynômes à coefficients complexes. Si l'égalité $P(A)=Q(A)$ entraîne $P=Q$, on dira que $A$ est un ensemble d'unicité généralisée (pour les polynômes). On construit dans cet article un sous-ensemble $A$ de $\mathbb{C}$ tel que $A$ et $\mathbb{C} \backslash A$ sont d'unicité généralisée, et on donne aussi l'exemple d'un ensemble d'unicité généralisée qui est ouvert, connexe et non borné.
\end{abstract}

Soit $A$ un sous-ensemble non vide du plan complexe $\mathbb{C}$; on dira que $A$ est un ensemble d'unicité généralisée pour les polynômes, en bref d'unicité généralisée, si l'égalité $P(A)=Q(A)$ pour deux fonctions polynômes $P$ et $Q$ de la variable complexe (i.e. si $P$ et $Q$ ont même image sur $A$ ) entraîne $P=Q$; le problème étant alors de trouver des ensembles qui sont ou ne sont pas d'unicité généralisée. H. Diamond, C. Pomerance, L. Rubel dans [1] ont étudié ce problème pour les fonctions entières: il résulte facilement des techniques utilisées là que, par exemple, les ensembles dénombrables $A_{s}=\left\{n^{s}: n \in \mathbb{N}^{*}\right\}$ (où $s \in \mathbb{R} \backslash\{0,1\}$ ), et $F_{\epsilon}=$ $\left\{p_{n}^{\epsilon}, n \in \mathbb{N}^{*}\right\}$ (où $\epsilon= \pm 1$ et $\left(p_{n}\right)$ est la suite des nombres premiers), sont d'unicité généralisée (pour les polynômes). D'autre part dans [2], E.H. Johnston construit un ouvert connexe borné qui est d'unicité généralisée pour les fonctions entières (et donc pour les polynômes). Dans cet article nous construisons un sous-ensemble $A$ de $\mathbb{C}$ tel que $A$ et $\mathbb{C} \backslash A$ sont des ensembles d'unicité généralisée (cf. le corollaire $\mathrm{du}$ théorème $\mathrm{A}$ ) et dans le théorème $\mathrm{B}$ nous donnons, en particulier, l'exemple d'un ensemble d'unicité généralisée qui est ouvert, connexe et non borné.

Notation. Si $A$ est un sous-ensemble non vide borné de $\mathbb{C}$, on note $|A|$ l'intervalle $[a, b]$ où $a=\inf \{|z|: z \in A\}$ et $b=\sup \{|z|: z \in A\}$.

1.

On commence par donner, dans le théorème qui suit, l'exemple d'un ensemble d'unicité généralisée non borné, non connexe et ouvert si on veut.

Received by the editors January 29, 1996 and, in revised form, October 1, 1996.

1991 Mathematics Subject Classification. Primary 30C10.

(C)1998 American Mathematical Society 
Théorème A. Soit $\left(r_{n}\right)_{n \geq 1},\left(R_{n}\right)_{n \geq 1}$ deux suites de réels strictement positifs telles que:

(i) $r_{n} \sim R_{n}(n \rightarrow \infty), r_{n} \leq R_{n}$ pour tout $n$.

(ii) $\lim _{n} \frac{r_{n+1}}{r_{n}^{s}}=+\infty$ pour chaque $s \geq 1$.

Soit $\left(\Omega_{n}\right)_{n \geq 1}$ une suite de sous-ensembles non vides bornés de $\mathbb{C}$ telle que:

a) $\left|\Omega_{n}\right| \subset\left[r_{n}, R_{n}\right]$ pour $n \geq 1$.

b) diamètre $\left(\Omega_{n}\right)=O\left(e^{-r_{n}}\right)$.

Alors $\Omega=\bigcup_{n \geq 1} \Omega_{n}$ est un ensemble d'unicité généralisée.

Par exemple les suites $\left(r_{n}\right)$ et $\left(R_{n}\right)$ définies par $r_{n}=2^{2^{2^{n}}}$ et $R_{n}=r_{n}+$ $2^{2^{n}}$ vérifient les hypothèses. Le théorème montre en particulier que l'ensemble dénombrable $\left\{2^{2^{2^{n}}}, n \geq 1\right\}$ est un ensemble d'unicité généralisée, résultat énoncé dans [1]. La démonstration est basée sur le lemme suivant:

Lemme 1. Soit $P, Q$ deux polynômes non constants de degré $p$ et $q$ respectivement et $k=\max (p, q)$. Il existe deux réels stritement positifs $\alpha, \beta$ ne dépendant que de $P$ et $Q$ tels que si $P(z)=Q(Z)$ on a:

(i) $S i|z| \geq 1,|Z| \geq 1$ alors

$$
|z-Z| \leq \alpha \min \left[|z|^{k},|Z|^{k}\right]
$$

(ii) Si $P \neq Q$, il existe $r_{0} \geq 1$ tel que si $|z| \geq r_{0},|Z| \geq r_{0}$ on a:

$$
|z-Z| \geq \frac{\beta}{\min \left[|z|^{k-1},|Z|^{k-1}\right]} .
$$

Preuve. Pour un polynôme $S$ de degré $s$ on a: $|S(z)| \leq a|z|^{s}$ pour tout $z \in \mathbb{C}$ avec $|z| \geq 1$, où $a$ est un réel ne dépendant que de $S$.

Soit $P$ et $Q$ deux polynômes non constants de degré $p$ et $q$ respectivement et $z$, $Z$ tels que $P(z)=Q(Z)$.

(i) Ecrivons le développement de Taylor du polynôme $w \rightarrow Q(w)-P(z)$ en puissance de $(w-z)$ :

$$
Q(w)-P(z)=Q(z)-P(z)+\sum_{k=1}^{q} \frac{Q^{(k)}(z)}{k !}(w-z)^{k} .
$$

Alors si $|Z-z| \geq 1$ on a:

$$
|Z-z|^{q} \leq\left|b_{q}\right|^{-1}\left(\sum_{k=1}^{q-1} \frac{\left|Q^{(k)}(z)\right|}{k !}+|P(z)-Q(z)|\right)|Z-z|^{q-1},
$$

où $b_{q}$ est le coefficient de degré $q$ de $Q$, et donc si $|z| \geq 1$

$$
|Z-z| \leq \alpha^{\prime}|z|^{k}
$$

$\alpha^{\prime}$ étant une constante ne dépendant que de $P$ et $Q$; on a alors aussi $|Z-z| \leq \alpha^{\prime \prime}|Z|^{k}$ pour $|Z| \geq 1$, où $\alpha^{\prime \prime}$ ne dépend que de $P$ et $Q$ et ceci donne (i).

(ii) Si $P \neq Q$, il existe $r_{0} \geq 1$ tel que $\inf \left\{|P(w)-Q(w)|,|w| \geq r_{0}\right\}=c>0$. En utilisant encore le développement (1), on a pour $|z| \geq r_{0}$ et si $|Z-z| \leq 1$ :

$$
0<c \leq|Q(z)-P(z)| \leq\left(\sum_{k=1}^{q} \frac{\left|Q^{(k)}(z)\right|}{k !}\right)|Z-z| \leq b|z|^{q-1}|Z-z|,
$$


où $b$ est une constante ne dépendant que de $Q$. En échangeant $z$ et $Z$ on obtient une inégalité du même type, et ceci entraîne (ii).

Preuve du Théorème $A$. Notons pour $n$ entier $\geq 2, \Delta_{n}=\left\{z:|z| \leq R_{n-1}\right\}, V_{n}=$ $\left\{z:|z| \geq r_{n+1}\right\}$ et $E_{n}=\Delta_{n} \cup V_{n}$. Soit alors deux polynômes $P, Q$ non constants de degré $p$ et $q$ respectivement et $k=\max (p, q)$; nous affirmons que $P\left(\Omega_{n}\right) \cap Q\left(E_{n}\right)=\emptyset$ (et donc aussi $Q\left(\Omega_{n}\right) \cap P\left(E_{n}\right)=\emptyset$ ) pour tout entier $n$ assez grand. En effet sinon on a l'une ou l'autre des possibilités suivantes.

- $P\left(\Omega_{n}\right) \cap Q\left(\Delta_{n}\right) \neq \emptyset$ pour une infinité de $n$; pour chacun de ces $n$, il existe $Z \in \Delta_{n}$ et $z \in \Omega_{n}$ tels que $P(z)=Q(Z)$ et, d'après (i) du lemme 1 ,

$$
|Z-z| \leq \alpha|Z|^{k} \text { et donc } 0 \leq r_{n}-R_{n-1} \leq \alpha R_{n-1}^{k},
$$

ce qui contredit les hypothèses (ii) du théorème $\mathrm{A}$,

- $P\left(\Omega_{n}\right) \cap Q\left(V_{n}\right) \neq \emptyset$ pour une infinité de $n$; alors comme précédemment avec $Z \in V_{n}$ et $z \in \Omega_{n}$ on a, vu (i) du lemme 1 :

$$
|Z-z| \leq \alpha|z|^{k} \text { et donc } 0 \leq r_{n+1}-R_{n} \leq \alpha R_{n}^{k},
$$

Supposons maintenant qu'il existe deux polynômes distincts $P$ et $Q$ tels que $P(\Omega)=Q(\Omega)$, alors $P$ et $Q$ sont non constants et vu ce qui précède, on a $P\left(\Omega_{n}\right)=$ $Q\left(\Omega_{n}\right)$ pour tout $n$ assez grand; pour chacun de ces $n$ il existe donc $z$ et $Z$ dans $\Omega_{n}$ tels que $P(z)=Q(Z)$, et d'après (ii) du lemme 1 pour tout $n$ assez grand on a:

$$
|Z-z| \geq \frac{\beta}{|z|^{k-1}} \text { et donc diamètre }\left(\Omega_{n}\right) \geq \frac{\beta}{R_{n}^{k-1}},
$$

ce qui contredit l'hypthèse b) du théorème $\mathrm{A}$.

Finalement on a $P(\Omega)=Q(\Omega)$ seulement si $P=Q$, et $\Omega$ est bien un ensemble d'unicité généralisée.

Remarque. La démonstration du théorème montre que l'ensemble $\Omega$ est encore un ensemble d'unicité généralisée si on remplace l'hypthèse b) par l'une ou l'autre des hypothèses suivantes:

- $\Omega_{n}$ est un ensemble d'unicité généralisée pour une infinité de valeurs de $n$.

- Diamètre $\left(\Omega_{n}\right) \leq M e^{-r_{n}}$ pour une infinité de valeurs de $n$, où $M$ est un réel indépendant de $n$.

Une conséquence de la démonstration du théorème $\mathrm{A}$ est le résultat suivant.

Corollaire. Il existe $A \subset \mathbb{C}$ tel que $A$ et $\mathbb{C} \backslash A$ sont des ensembles d'unicité généralisée.

Preuve. Gardons les notations et les hypothèses du théorème $\mathrm{A}$ et soit, par exemple, pour chaque entier $n \geq 1$ l'ensemble $\Omega_{n}^{\prime}$ défini par:

$$
\Omega_{n}^{\prime}= \begin{cases}\Omega_{3 n-1} \cup \Omega_{3 n} \cup \Omega_{3 n+1} & \text { si } n \text { est impair, } \\ A_{R_{3 n-2}, R_{3 n+1}} \backslash\left(\Omega_{3 n-1} \cup \Omega_{3 n} \cup \Omega_{3 n+1}\right) & \text { si } n \text { est pair, }\end{cases}
$$

où, pour $0 \leq r \leq R, A_{r, R}=\{z: r \leq|z| \leq R\}$. Alors l'ensemble $A=\bigcup_{n \geq 1} \Omega_{n}^{\prime}$ (et donc aussi $\mathbb{C} \backslash A$ ) est d'unicité généralisée, puisque si $P(A)=Q(A)$ pour deux polynômes $P$ et $Q$, la démonstration du théorème A montre que $P\left(\Omega_{3 n}\right)=Q\left(\Omega_{3 n}\right)$ pour tout entier $n$ impair assez grand, et on conclut comme précédemment que, si $P \neq Q$, cette égalité est impossible vu l'hypothèse b) sur le diamètre des $\Omega_{3 n}$. 


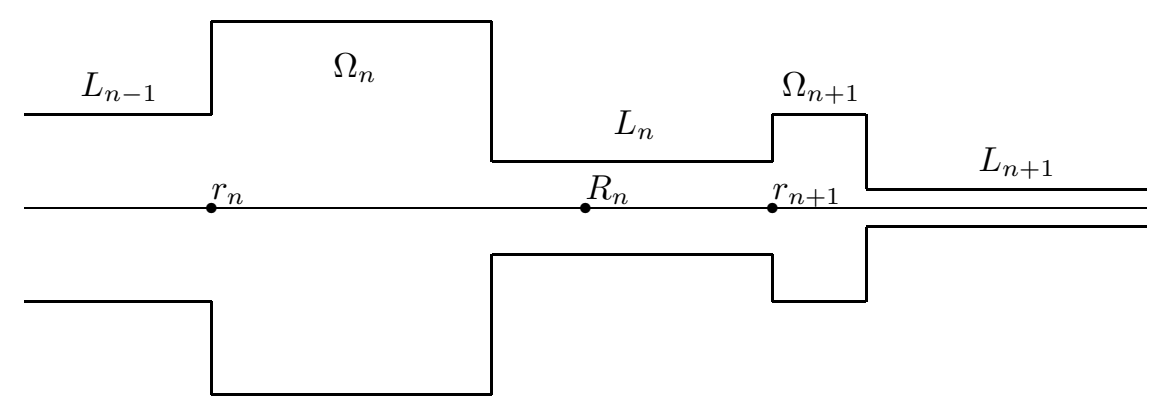

2.

Nous donnons dans ce paragraphe le résultat principal de cet article.

Théorème B. Soit $\left(r_{n}\right),\left(R_{n}\right)$ deux suites de reéls vérifiant les hypothèses du théorème A. Soit $\left(\Omega_{n}\right)$ et $\left(L_{n}\right)$ deux suites de sous-ensembles non vides mesurables de $\mathbb{C}$ tels que:

a) $\left|\Omega_{n}\right| \subset\left[r_{n}, R_{n}\right],\left|L_{n}\right| \subset\left[r_{n}, R_{n+1}\right]$ pour $n \geq 1$.

b) diamètre $\left(\Omega_{n}\right)=O\left(e^{-r_{n}}\right)$.

c) aire $\left(\Omega_{n}\right) \geq \lambda e^{-2 r_{n}}$ pour tout $n$ assez grand, où $\lambda>0$ est un réel indépendant de $n$.

d) $\operatorname{aire}\left(L_{n}\right)=O\left(e^{-r_{n+2}}\right)$.

Alors l'ensemble $\Omega=\bigcup_{n \geq 1}\left(\Omega_{n} \cup L_{n}\right)$ est un ensemble d'unicité généralisée.

En choisant convenablement pour chaque entier $n, \Omega_{n}$ et $L_{n}$ ouverts et connexes, on obtient facilement un ensemble $\Omega$ ouvert et connexe. La figure ci-dessus illustre ceci dans un cas particulier: $\Omega_{n}$ est un rectangle ouvert de diamètre $e^{-r_{n}}$ et $L_{n}$ une bande ouverte qui relie $\Omega_{n}$ à $\Omega_{n+1}$.

Remarque. D'après le corollaire du théorème de la section 2 de [2], l'ensemble de l'exemple ci-dessus n'est pas d'unicité généralisée pour les fonctions entières.

Preuve. Supposons qu'il existe deux polynômes distincts $P$ et $Q$ tels que $P(\Omega)=$ $Q(\Omega)$. On a vu dans la preuve du théorème A que $P\left(\Omega_{n}\right) \cap Q\left(E_{n}\right)=\emptyset$ pour tout $n$ assez grand; d'autre part si $P\left(\Omega_{n}\right) \cap Q\left(\Omega_{n}\right) \neq \emptyset$ pour une infinité de valeurs de $n$, on aurait une contradiction avec l'hypothèse sur le diamètre de $\Omega_{n}$ en procédant comme dans la fin de la preuve du théorème A: c'est donc que $P\left(\Omega_{n}\right) \cap Q\left(\Omega_{n}\right)=\emptyset$ et que

$$
P\left(\Omega_{n}\right) \subset Q\left(L_{n-1} \backslash E_{n}\right) \cup Q\left(L_{n} \backslash E_{n}\right) \subset Q\left(L_{n-1}\right) \cup Q\left(L_{n}\right)
$$

pour tout $n$ assez grand.

La comparaison des aires des membres extrêmes de (2), à l'aide du lemme qui suit, va montrer que ces inclusions sont impossibles.

Lemme 2. Soit $S$ un polynôme non constant de degré s. Alors pour tout $r$ assez grand et pour tout sous-ensemble mesurable borné de $\mathbb{C}$ avec $|E|=[r, R]$ on a:

$$
\operatorname{ar}^{2(s-1)} \text { aire }(E) \leq \operatorname{aire} S(E) \leq b R^{2(s-1)} \text { aire }(E)
$$

où a et b sont des réels strictement positifs ne dépendant que de $S$. 
Preuve. (i) Commençons par démontrer la seconde inégalité, et pour cela considérons dans un premier temps un disque $\Delta$ de rayon $\rho$. Alors, d'après le théorème des accroissements finis, pour $z, w$ dans $\Delta$ on a:

$$
|S(z)-S(w)| \leq \sup _{t \in \Delta}\left|S^{\prime}(t)\right||z-w|
$$

et donc le diamètre de $S(\Delta)$ est inférieur ou égal à $2 \sup _{t \in \Delta}\left|S^{\prime}(t)\right| \rho$, ce qui conduit à l'inégalité

$$
\operatorname{aire}(S(\Delta)) \leq 4 \sup _{t \in \Delta}\left|S^{\prime}(t)\right|^{2} \text { aire }(\Delta) \text {. }
$$

Maintenant soit $K$ un compact de $\mathbb{C}$; on peut alors recouvrir $K$ par un nombre fini de disques $\Delta_{1}, \ldots, \Delta_{n}$ tels que:

$$
\sum_{i=1}^{n} \operatorname{aire}\left(\Delta_{i}\right) \leq 2 \operatorname{aire}(K), \sup _{t \in \bigcup_{i=1}^{n} \Delta_{i}}\left|S^{\prime}(t)\right| \leq 2 \sup _{t \in K}\left|S^{\prime}(t)\right| .
$$

Puisque aire $(S(K)) \leq \sum_{i=1}^{n}$ aire $\left(S\left(\Delta_{i}\right)\right)$ on obtient en utilisant (3) et (4):

$$
\operatorname{aire}(S(K)) \leq 16 \sup _{t \in K}\left|S^{\prime}(t)\right|^{2} \operatorname{aire}(K) .
$$

Finalement soit $E$ un sous-ensemble mesurable borné de $\mathbb{C}$; on peut écrire $E=$ $\left(\bigcup_{n} K_{n}\right) \cup N$ où $\left(K_{n}\right)$ est une suite croissante de compacts et $N$ un ensemble d'aire nulle; puisque $S(N)$ est aussi d'aire nulle on obtient en utilisant (5):

$$
\operatorname{aire}(S(E)) \leq 16 \sup _{t \in E}\left|S^{\prime}(t)\right|^{2} \operatorname{aire}(E)
$$

Si $|E| \subset[0, R]$ on en déduit la seconde inégalité du lemme.

(ii) Pour la première inégalité du lemme nous allons utiliser la formule suivante, dont nous donnons une démonstration succincte dans la section 3: soit $\Omega_{1}, \Omega_{2}$ deux ouverts connexes de $\mathbb{C}$ et $f$ une application holomorphe propre de $\Omega_{1}$ dans $\Omega_{2}$ de multiplicité $m$, alors pour tout $g \in L^{1}\left(\Omega_{2}\right)$ on a:

$$
\int_{\Omega_{1}} g(f(z))\left|f^{\prime}(z)\right|^{2} d z=m \int_{\Omega_{2}} g(w) d w
$$

où on intègre par rapport à la mesure de Lebesgue du plan. Ceci appliqué à $\Omega_{1}=$ $\Omega_{2}=\mathbb{C}, f=S, g$ la fonction caractéristique de $S(E)$ donne:

$$
\int_{-1(S(E))}\left|S^{\prime}(z)\right|^{2} d z=s \text { aire }(S(E))
$$

et aussitôt les inégalités:

$$
\text { s aire } \left.S(E) \geq \int_{E} \mid S^{\prime}(z)\right)\left.\right|^{2} d z \geq \inf _{z \in E}\left|S^{\prime}(z)\right|^{2} \operatorname{aire}(E) .
$$

Si $|E| \subset[r,+\infty)$ avec $r$ assez grand, la première inégalité du lemme en résulte.

Fin de la démonstration du Théorème $B$. l'hypothèse d), il existe $M>0$ tel que aire $\left(L_{n}\right) \leq M e^{-r_{n+2}}$ pour tout $n$. L'inclusion (2) conduit à l'inégalité sur les aires:

$$
\operatorname{aire}\left(P\left(\Omega_{n}\right)\right) \leq \operatorname{aire}\left(Q\left(L_{n-1}\right)\right)+\operatorname{aire}\left(Q\left(L_{n}\right)\right) .
$$

Le lemme 2 donne alors:

$$
\begin{gathered}
\operatorname{aire}\left(P\left(\Omega_{n}\right)\right) \geq a r_{n}^{2(p-1)} \lambda e^{-2 r_{n}} \geq a \lambda e^{-2 r_{n}}, \\
\operatorname{aire}\left(Q\left(L_{n-1}\right)\right)+\operatorname{aire}\left(Q\left(L_{n}\right)\right) \leq 2 b M R_{n+1}^{2(q-1)} e^{-r_{n+1}}
\end{gathered}
$$


pour tout $n$ assez grand, et on a donc, puisque $r_{n} \sim R_{n}$ :

$$
r_{n+1}^{2(1-q)} \exp \left(r_{n+1}\left(1-\frac{2 r_{n}}{r_{n+1}}\right)\right)=O(1),
$$

ce qui est impossible puisque $\lim _{n} r_{n} / r_{n+1}=0$, et ceci termine la démonstration $\mathrm{du}$ théorème $\mathrm{B}$.

Le théorème qui suit est certainement connu, et l'auteur ne prétend à aucune originalité; il pourrait s'intituler: Formule du changement de variable dans les intégrales via une application holomorphe propre.

Théorème C. Soit $\Omega_{1}, \Omega_{2}$ deux ouverts connexes de $\mathbb{C}$, et $f$ une application holomorphe propre de multiplicité $m$ de $\Omega_{1}$ dans $\Omega_{2}$. Alors on a la formule:

$$
m \int_{\Omega_{2}} g(\omega) d \omega=\int_{\Omega_{1}} g(f(z))\left|f^{\prime}(z)\right|^{2} d z \quad \forall g \in L^{1}\left(\Omega_{2}\right) .
$$

Si $m=1$, on retrouve la formule du changement de variable habituelle, puisque dans ce cas $f$ est une bijection holomorphe, et donc un difféomorphisme, de $\Omega_{1}$ sur $\Omega_{2}$.

Preuve. On suppose connu les résultats élémentaires sur les applications holomorphes propres: voir, par exemple, W. Rudin, Function Theory in the Unit Ball in $\mathbb{C}^{n}$, Spinger-Verlag (1980), Ch.XV. Nous avons besoin essentiellement des résultats suivants: notons $Z$ l'ensemble des zéros de $f^{\prime}$ dans $\Omega_{1} ; Z$ est au plus dénombrable et n'a pas de point d'accumulation dans $\Omega_{1}$ et il en est de même de $W=f(Z)$ dans $\Omega_{2}$; pour chaque $\omega \in \Omega_{2} \backslash W, f^{-1}(\omega)=\left\{z_{1}, \cdots, z_{m}\right\}\left(z_{i} \neq z_{j}\right.$ pour $\left.i \neq j\right)$ et il existe $N$ ouvert contenant $\omega, D_{1}, \cdots, D_{m}$ ouverts contenant respectivement $z_{1}, \cdots, z_{m}$ tels que $\bar{D}_{i} \cap \bar{D}_{j}=\emptyset$ pour $i \neq j, f_{\mid D_{i}}$ est une bijection de $D_{i}$ sur $N$ pour chaque $i=1, \cdots, m$ et $f^{-1}(N)=\bigcup_{1 \leq i \leq m} D_{i}$.

Soit alors $g \in L^{1}\left(\Omega_{2}\right)$ dont le support est inclus dans $N$. En utilisant la formule de changement de variable habituelle on obtient:

$$
\int_{\Omega_{2}} g(\omega) d \omega=\int_{N} g(\omega) d \omega=\int_{D_{i}} g(f(z))\left|f^{\prime}(z)\right|^{2} d z
$$

pour chaque $i=1,2, \cdots, m$, et donc

$$
m \int_{\Omega_{2}} g(\omega) d \omega=\int_{D} g(f(z))\left|f^{\prime}(z)\right|^{2} d z=\int_{\Omega_{1}} g(f(z))\left|f^{\prime}(z)\right|^{2} d z
$$

où $D=\bigcup_{1 \leq i \leq m} D_{i}$. De (6) on déduit la formule du théorème dans le cas général par un argument standard utilisant une partition de l'unité.

\section{RÉFÉRENCES}

1. Diamond, H; Pomerance, C; Rubel, L. Sets on which an entire functions is determined by its range. Math. Z, 176, 383-398 (1981). MR 82e:30031

2. Johnston, E.H. On sets of range uniqueness. Math. Z, 184, 533-547 (1983). MR 85b:30038

Département de Mathématiques, case 051, Université Montpellier II, Place Eugène Bataillon, 34095 Montpellier, France

E-mail address: savoyant@math.univ-montp2.fr 